\title{
Diffraction contrast analysis of dislocations in 2D materials using true dark-field and 4D-STEM in SEM
}

Peter Denninger ${ }^{1}$, Peter Schweizer ${ }^{2}$, Christian Dolle $^{3}$ and Erdmann Spiecker ${ }^{1}$

${ }^{1}$ Institute of Micro- and Nanostructure Research (IMN) \& Center for Nanoanalysis and Electron Microscopy (CENEM), Interdisciplinary Center for Nanostructured Films (IZNF), Department of Materials Science and Engineering, Friedrich-Alexander University Erlangen-Nürnberg (FAU), Erlangen, Germany, Erlangen, Bayern, Germany, ${ }^{2}$ Empa, Thun, Switzerland, ${ }^{3}$ Karlsruher Institut für Technologie, Karlsruhe, Baden-Wurttemberg, Germany

Diffraction techniques are ubiquitous in electron microscopy, especially in Transmission Electron Microscopy (TEM). TEM enables the use of an objective aperture placed in the diffraction plane to increase contrast in bright-field or dark-field mode. On the other hand, Scanning Electron Microscopy (SEM) also offers several diffraction techniques, such as EBSD and TKD. However, the exploration of on-axis transmission diffraction is still in its infancy. In recent years there have been several developments in this direction [1-3] to take advantage of the unique features of SEM, such as low acceleration voltages, large chamber size, and strong topographic contrast. However, further developments to also include an aperture system for dedicated diffraction contrast imaging have not been realized so far.

The characterization of crystal dislocations is certainly the most prominent example of diffraction contrast analysis and is traditionally performed in TEM using bright field or dark field imaging under two-beam condition. Alternatively, diffraction contrast in STEM mode can also be applied for dislocation imaging and has become quite popular, as it produces a more uniform dislocation contrast which is less affected by foil bending. However, for 2D materials the situation changes. First, the diffracted beams show very low intensities at the high electron energies typically used in TEM-STEM. Second, two-beam conditions can hardly be realized since always several $\mathbf{g}$ vectors are excited due to extended streaking in reciprocal space. Therefore, diffraction contrast imaging under well-defined conditions can only be realized in darkfield mode. At lower electron energies typically used in SEM instruments $(1 \ldots 30 \mathrm{keV})$ the strength of elastic scattering is strongly increased providing ideal conditions for dark-field imaging. In order to perform a diffraction contrast analysis of dislocations, e.g. for determination of Burgers vectors, individual reflections have to be isolated and directed onto the STEM detector. Even though the latter is typically segmented only a small movable aperture placed between sample and detector enables reliable and flexible selection of individual reflections $\mathbf{g}$ for dark field imaging. To be able to place the aperture an on-axis transmission diffraction mode is required.

A simple solution to on-axis transmission diffraction in SEM is our low-energy nanodiffraction (LEND) setup, which is based on the combination of a fluorescent screen positioned below the sample with a dedicated CMOS camera (Fig. 1a,b). The technique has been implemented and successfully tested for several material systems [3]. As example, LEND patterns of graphene (at $5 \mathrm{keV}$ ) and of polycrystalline $\mathrm{Au}($ at $10 \mathrm{keV})$ are depicted in Fig. 1c. These patterns look very much like diffraction patterns in TEM and contain the same rich information on the scattering behavior and crystal structure of the specimen. For ultrathin specimens like 2D meterials, the spatial resolution of LEND is only limited by the probe size.

Based on the LEND technique we insert an aperture within the transmitted beam paths in such a way, that all electrons are blocked except one (freely chosen) diffracted beam, which passes to the STEM detector (Fig. 1b). Upon scanning a "true" dark-field (DF) image is generated, which is directly comparable to dark 
field images obtained in TEM. The apertures are custom-made out of thin metal sheets using a laser cutting device. This enables a rational design of aperture shapes tailor-made for specific applications (Fig. 1d). The navigation and adjustment of the aperture are performed with a piezo-controlled micromanipulator that offers a precise movement in all dimensions with sub-nm resolution.

To showcase the power of this approach Fig. 2 compares DF images of basal dislocations in bilayer graphene taken in SEM at $20 \mathrm{kV}$ and in TEM at $80 \mathrm{kV}$, respectively. The two image sets look very similar and show comparable resolution, however, the acquisition time in SEM was considerably lower than in TEM due to the stronger scattering and thus increased DF intensity at low voltages. In order to determine the Burgers vectors of the dislocations three DF images were acquired using three different $\{11-20\}$ reflections contained in the [0001] zone axis. The direction of the Burgers vector can be identified by application of the $\mathbf{g} \times \mathbf{b}=0$ invisibility criterion, which describes the invisibility of dislocation if the Burgers vector $\mathbf{b}$ is perpendicular to the selected diffraction vector $\mathbf{g}$. As can be seen from Fig. 2 the true DF STEM technique combined with LEND in SEM reveals the same Burgers vectors as DF TEM and is therefore well suited for reliable Burgers vector analysis of dislocations in 2D-materials. A closer analysis shows that even the sign of the Burgers vector can be determined by analyzing DF images taken under sample tilt, as we demonstrated for the TEM analysis in [4].

A different way to acquire DF images is the acquisition of complete diffraction mappings, also known as 4D-STEM, where the electron beam scans over the sample in a 2D pixel array. For each pixel, a twodimensional diffraction pattern is acquired (hence 4D) and the resulting 4D-STEM dataset stack contains a huge amount of information [5]. While 4D-STEM has been developed in TEM-STEM instruments the technique can be equally applied in SEM with the same advantages for 2D materials mentioned above [3]. A simple data analysis is the reconstruction of DF images by placing a virtual aperture around certain diffraction spots. While 4D-STEM gives us unparalleled local information, the limiting factors for the technique are acquisition time (or resolution) and dose.

In our contribution we will compare "true" DF STEM and 4D-STEM in SEM for analysis of dislocations in 2D materials. Moreover, we will discuss strategies for simultaneous acquisition of diffraction patterns and BF/DF images in SEM and their potential use for in situ studies. [6] 

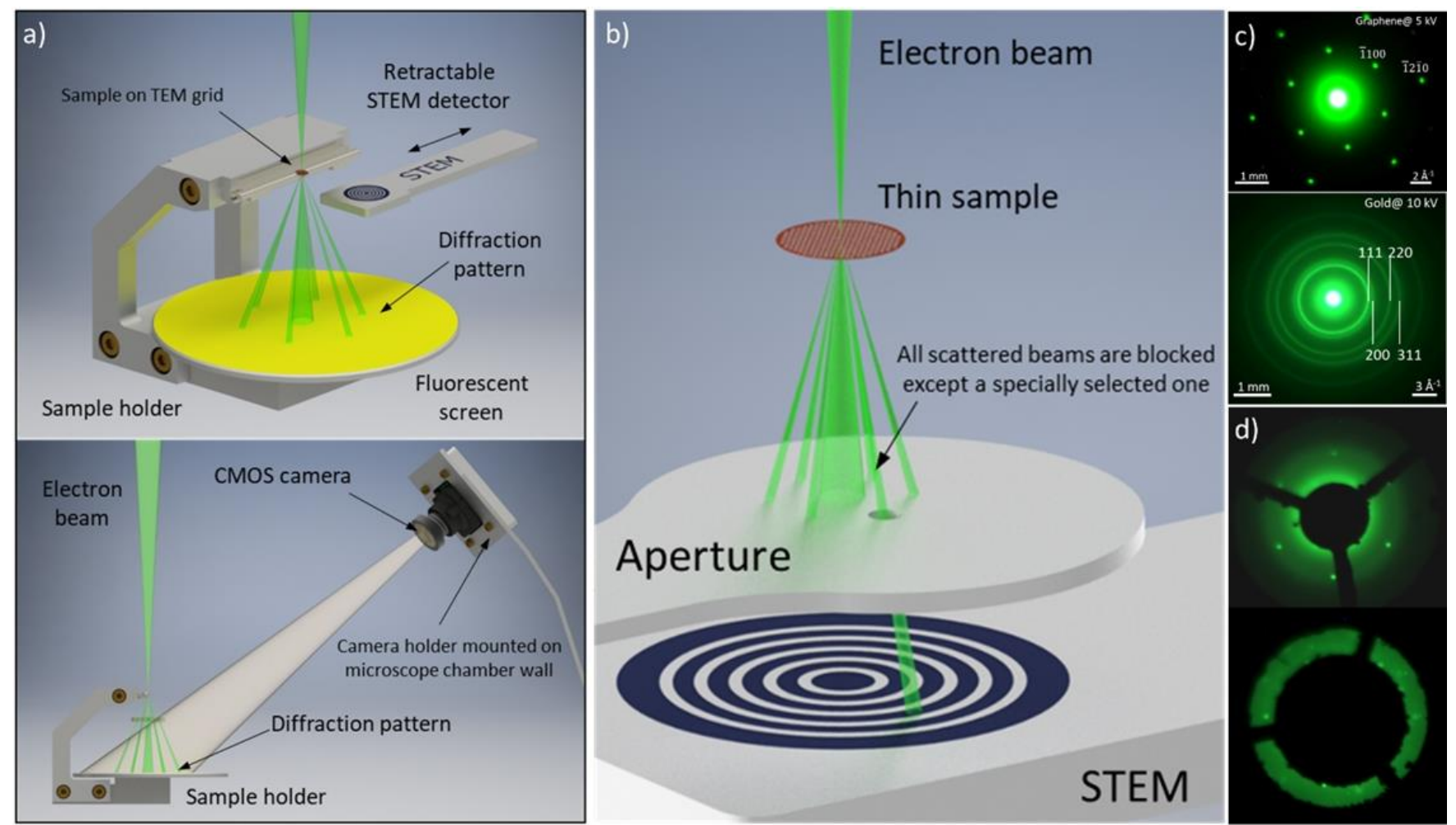

Figure 1. Diffraction in the SEM. a) Setup of Low Energy Nano Diffraction (LEND). b) Concept of the true dark-field technique. c) Diffraction patterns of monolayer graphene and polycrystalline gold film. d) Diffraction patterns with inserted, custom-shaped apertures.
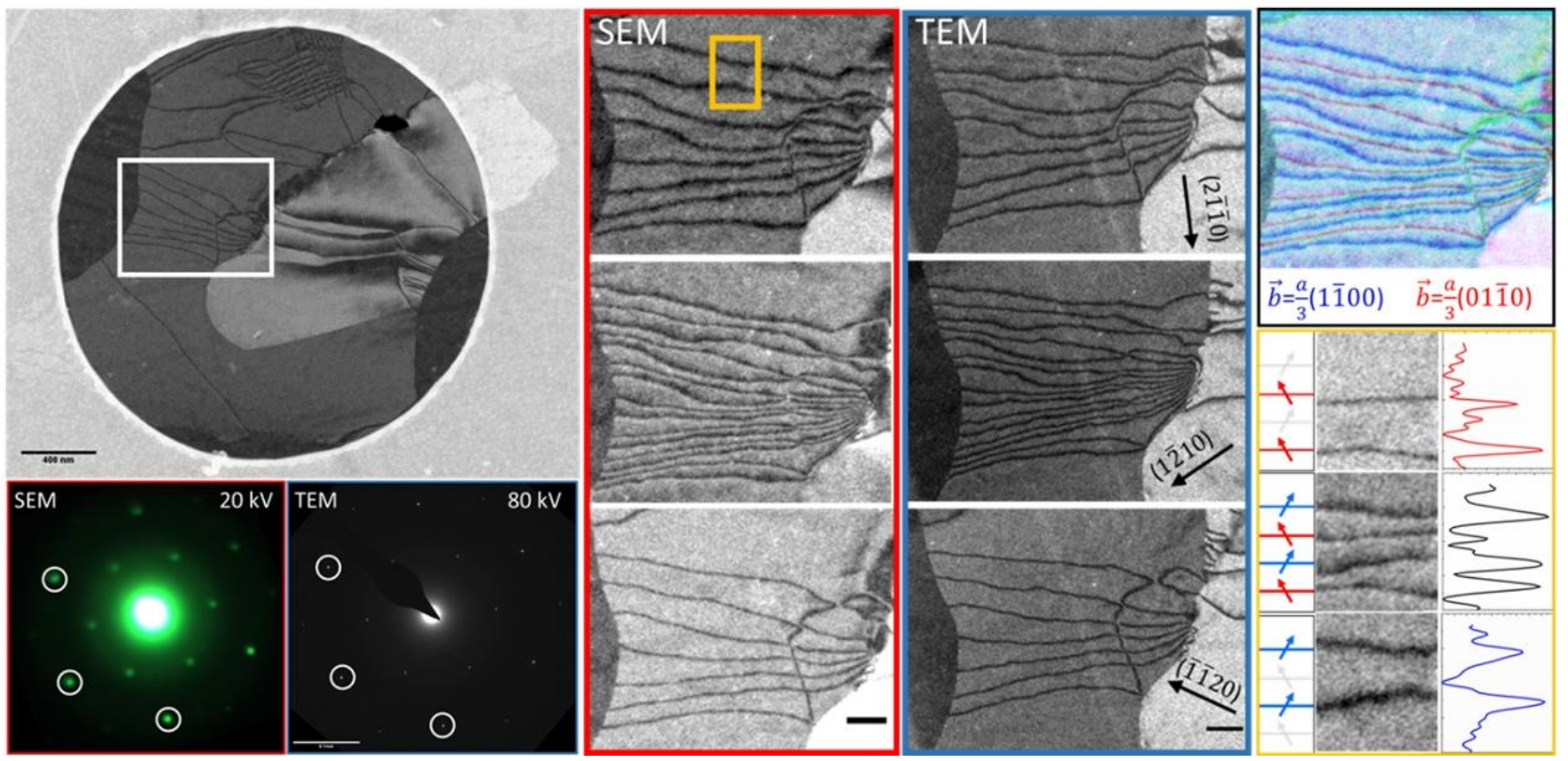

Figure 2. Diffraction analysis of basal dislocations in bilayer graphene using true dark-field imaging in SEM (20 kV, red) and, for comparison, conventional dark-field imaging in TEM ( $80 \mathrm{kV}$, blue). The two techniques produce comparable images and, based on the invisibility criterion, deliver the same dislocation Burgers vectors. 


\section{References}

[1] C. Sun, E. Muller, M. Meffert, D. Gerthsen, Analysis of crystal defects by scanning transmission electron microscopy (STEM) in a modern scanning electron microscope, Adv. Struct. Chem. Imaging 5 (2019) 1.

[2] B.W. Caplins, J.D. Holm, R.R. Keller, Transmission imaging with a programmable detector in a scanning electron microscope, Ultramicroscopy 196 (2019) 40-48.

[3] P. Schweizer, P. Denninger, C. Dolle, E. Spiecker, Low energy nano diffraction (LEND) - A versatile diffraction technique in SEM, Ultramicroscopy 213 (2020) 112956.

[4] P. Schweizer, C. Dolle, E. Spiecker, In situ manipulation and switching of dislocations in bilayer graphene, Science Advances 4 (2018), p. eaat4712P.

[5] C. Ophus, P. Ercius, M. Sarahan, C. Czarnik, J. Ciston, Recording and using 4D-STEM datasets in materials science, Microsc. Microanal. 20 (2014) 62-63.

[6] We gratefully acknowledge financial support by the German Research Foundation (DFG) within the frameworks of the research training group GRK1896 "In situ Microscopy with Electrons, X-rays and Scanning Probes" and the Collaborative Research Centre 953 "Synthetic C-allotropes". 